\title{
Análisis de la mejora del aprendizaje tras la incorporación de metodologías activas en una asignatura optativa del Grado de Ingeniería Química
}

\author{
María Sancho Fernández ${ }^{\mathrm{a}}$, Beatriz García-Fayos ${ }^{\mathrm{b}}$ y José Miguel Arnal Arnal ${ }^{\mathrm{c}}$ \\ ${ }^{a}$ Universitat Politècnica València, msanchof@iqn.upv.es ${ }^{b}$ Universitat Politècnica València, \\ beagarfa@iqn.upv.es y ${ }^{c}$ Universitat Politècnica València, jarnala@iqn.upv.es
}

\begin{abstract}
Adaptation to the European Higher Education Area has required the implementation of active methodologies that promote the involvement of students in their own learning process. This type of methodologies are particularly suitable for optional subjects of last courses, as they usual are attended by a low number of students and this situation gives the possibility of giving them a multidisciplinary approach. In the academic year 2013-14, a new optional subject called: "Industrial Safety" was implemented in the last year of the degree in Chemical Engineering. In that first course, teaching was performed by traditional methodology with master classes, producing unsatisfactory academic results. For this reason, in the following academic year (2014-15) we designed new activities based on different active methodologies, in order to improve the subject, in academic results and satisfaction of students. This work analyses the improvement of the learning in several key contents of the subject, based on the responses in the initial diagnostic survey, and final marks in the evaluation items corresponding to each content. The results show a significant improvement in learning of most of the contents and excellent final academic results, which seem to be favored by the new activities and implemented methodologies.
\end{abstract}

Keywords: Active methodologies, learning, improvement, safety, Chemical Engineering

\footnotetext{
Resumen

La adaptación al Espacio Europeo de Educación Superior ha requerido la implementación de metodologías activas que promuevan la implicación de los estudiantes en su propio proceso de aprendizaje. Este tipo de metodologías son especialmente adecuadas para asignaturas optativas de últimos cursos, por su menor número de alumnos y por la posibilidad de darles un enfoque multidisciplinar. En el curso académico 2013-14, con la implantación del último curso del Grado en Ingeniería Química, se puso en marcha una nueva asignatura optativa: "Seguridad Industrial". En ese primer curso, se siguió la metodología tradicional con lecciones magistrales, con resultados finales poco satisfactorios. Por ello, en el siguiente curso académico (2014-15) se diseñaron nuevas actividades, basadas en distintas
} 
metodologías activas, con el fin de mejorar la asignatura, tanto desde el punto de vista académico como de satisfacción de los alumnos. Este trabajo analiza la mejora del aprendizaje de los estudiantes en distintos contenidos clave de la asignatura, a partir de las respuestas de la encuesta inicial de diagnóstico pasada a los alumnos, y de sus calificaciones en los actos de evaluación correspondientes a dichos contenidos. Los resultados muestran una mejora significativa en el aprendizaje en la mayoría de contenidos y unos excelentes resultados finales, que parecen estar favorecidos por las actividades y metodologías implementadas.

Palabras clave: Metodologías activas, aprendizaje, mejora, seguridad, Ingeniería Química

\section{Introducción}

La adaptación al Espacio Europeo de Educación Superior ha requerido la implantación de metodologías activas para promover la implicación de los estudiantes en su propio proceso de aprendizaje. Simultáneamente, las metodologías activas facilitan la formación en determinadas competencias transversales y permiten un acercamiento del estudiante a su futura labor profesional. Además, existen ya varios estudios que han demostrado que la aplicación de metodologías activas mejora significativamente los resultados académicos de los estudiantes (Palazón-Pérez De Los Cobos, 2011). Por todo ello, en un futuro próximo, el proceso de enseñanza-aprendizaje tendrá lugar de manera más probable a través de una combinación de proyectos, problemas, diseño, desarrollo, creatividad, reflexión, etc (Badía \& García, 2006). En general, estas metodologías son más fáciles de aplicar en asignaturas optativas, por el menor número de alumnos que las cursan frente a las asignaturas troncales, $\mathrm{y}$ en los últimos cursos de las titulaciones, por el enfoque multidisciplinar que se puede aplicar.

Esta situación de necesaria implicación del estudiante y de acercamiento a su futuro laboral, es si cabe más imprescindible en determinadas materias o asignaturas con una relevancia significativa dentro de la labor profesional de los egresados. Este es el caso de la asignatura "Seguridad Industrial", una optativa de $4^{\circ}$ curso del Grado en Ingeniería Química (GIQ), en la que los estudiantes deben aprender a implementar la seguridad en los procesos de la industria química, siguiendo los procedimientos y regulaciones vigentes en cada momento.

A la vista de este planteamiento, el curso pasado se decidió incorporar metodologías activas en dicha asignatura, que había sido impartida por primera vez con la puesta en marcha del $4^{\circ}$ curso de GIQ (curso 2013-14), pero a través de lecciones magistrales convencionales, habiéndose obtenido resultados académicos poco satisfactorios.

Estos resultados poco gratificantes, junto con la necesidad de acercar la asignatura a la realidad profesional, hizo que se implementaran nuevas metodologías y actividades en el pasado curso 2014-15. De entre las posibles metodologías activas, se seleccionaron como más adecuadas para la asignatura las siguientes (García-Fayos, 2015):

(cc) EY-NC-ND 2016, Universitat Politècnica de València 
- Estudio de casos. En el método del caso, los alumnos resuelven situaciones más o menos complejas de acercamiento a la realidad profesional, mediante el análisis de información, el intercambio de opiniones y la toma de decisiones (Aramendi, 2014). Esta metodología permite trabajar en mayor profundidad los contenidos, así como reflexionar y analizar distintos aspectos relacionados con los mismos, existiendo varias experiencias de aplicación con éxito en asignaturas relacionadas con la seguridad en la industria química (Ferjencik, 2007 \& Shallcross, 2013). En la asignatura objeto de este trabajo, el estudio de casos se ha aplicado en prácticamente todas las sesiones teóricas, en las que se han analizado accidentes industriales para determinar las posibles causas de los mismos y proponer medidas para evitarlos.

- Role-playing. Esta metodología, en la que los estudiantes actúan con un papel determinado dentro del contexto laboral, consigue aumentar su interés e implicación al sentir que están trabajando en un caso real (Westrup, 2013), y ha sido aplicada con buenos resultados en asignaturas relacionadas con seguridad (Darbra, 2012 \& Graells, 2007). En el caso de la asignatura que nos ocupa, se ha incorporado esta metodología en algunas de las actividades diseñadas, de manera que los estudiantes han debido actuar como inspectores de seguridad o técnicos de prevención para analizar, siguiendo un procedimiento similar al que se realiza profesionalmente, distintas instalaciones, equipos y puestos de trabajo.

- Aprendizaje cooperativo. Se trata de una metodología en la que los estudiantes trabajan en grupo para realizar un actividad académica, que tiene como principales ventajas, frente al trabajo competitivo e individual: promover una actitud positiva frente al aprendizaje, conseguir un mejor entendimiento del propio aprendizaje y favorecer relaciones interpersonales positivas. Además, el aprendizaje cooperativo permite desarrollar algunas competencias transversales como el trabajo en grupo, el pensamiento crítico, la toma de decisiones y la resolución de conflictos (Barbera-Ribera, 2015). Todos los estudios experimentales sobre este tipo de aprendizaje coinciden en que se consigue un aprendizaje más profundo y duradero (Gil, 2007). Prácticamente en todas las actividades realizadas, y en paralelo a las metodologías anteriormente comentadas, se ha trabajado en grupos para fomentar la participación de los alumnos y desarrollar ciertas habilidades relacionadas con el trabajo en equipo, como la discusión y la toma de decisiones.

Las principales actividades diseñadas realizadas mediante la aplicación de las metodologías anteriormente descritas fueron las siguientes (García-Fayos, 2015):

(1) Análisis de accidentes. En la mayoría de sesiones de clase se visionaron uno o varios vídeos relacionados con los contenidos de dicha sesión, para que los estudiantes analizaran de manera cooperativa las posibles causas de los accidentes y propusieran medidas preventivas para haberlos evitado.

(2) Inspección de seguridad a un laboratorio. En esta actividad los estudiantes actuaron como inspectores (role-playing) de los laboratorios en los que realizan la 
mayor parte de las prácticas de la titulación. Además, la sesión de inspección se hace coincidir con una práctica de laboratorio de los alumnos de $3^{\text {er }}$ curso, que ellos ya realizaron en su momento, para analizar también los procedimientos de trabajo y los equipos empleados por sus compañeros. Posteriormente se lleva a cabo una sesión en aula de puesta en común de la actividad.

(3) Identificación y evaluación de riesgos, y propuesta de medidas preventivas. Esta actividad se desarrolla en tres sesiones (dos de aula y una de práctica informática). La actividad se basa en un role-playing en el que profesores y alumnos representan el papel de trabajadores de una misma empresa, y el resto de estudiantes actúan como técnicos de prevención para entrevistar a dicho trabajadores y obtener la información necesaria que les permita identificar y evaluar los riesgos de cada puesto de trabajo. Posteriormente, se realiza un nueva dinámica en la que, de forma cooperativa, se proponen las medidas preventivas adecuadas a los riesgos identificados.

(4) Aplicación del Reglamento de Almacenamiento de Productos Químicos a una sustancia inflamable. Para facilitar la comprensión y aplicación de los contenidos a impartir, se ha escogido como hilo conductor de la asignatura el proceso de producción de aceite de oliva, que los alumnos han estudiado previamente con detalle en una asignatura troncal del cuatrimestre anterior, impartida además por una de las profesoras de esta asignatura. En dicho proceso industrial se utilizan varias sustancias peligrosas que se rigen por este Reglamento, por lo que se aprovecha este contexto para diseñar el almacenamiento de una de ellas de acuerdo a la legislación.

(5) Visitas a empresa. Se realizaron dos visitas a empresa, una al principio de la asignatura para que los alumnos se iniciaran en la identificación de riesgos de una industria real; y la otra al final de la asignatura para que vean implementada de forma correcta la seguridad que han estudiadodurante la asignatura. En la primera visita los estudiantes tuvieron que actuar de nuevo como inspectores de seguridad e identificar riesgos en la planta.

La evaluación de la asignatura comprende las siguientes partes y porcentajes:

- Informes de actividades (40\%). Esta nota corresponde a cuatro informes escritos que deben entregar tras algunas de las actividades realizadas, como las inspecciones a laboratorio y empresa, y la práctica informática de evaluación de riesgos.

- Trabajo final (30\%). Los alumnos deben entregar al final de la asignatura un trabajo escrito de implementación de las medidas de seguridad en el proceso de producción de aceite de oliva, que además deben presentar oralmente. La parte escrita supone un $20 \%$ de la nota, y la parte oral un $10 \%$.

- Examen escrito (30\%). Se realiza un examen de respuesta abierta sobre los principales contenidos trabajados en la asignatura.

(c) EY-NC-ND 2016, Universitat Politècnica de València 


\section{Objetivos}

En un trabajo anterior se presentó la valoración de los estudiantes de los contenidos y actividades de la asignatura, y su percepción del grado de aprendizaje adquirido en determinadas competencias (García-Fayos, 2015).

Este trabajo complementa al anterior, ya que analiza la mejora del aprendizaje de los alumnos en la asignatura tras la implantación de las metodologías activas, a través de la comparación de los conocimientos mostrados al inicio de la misma y de los resultados finales alcanzados.

\section{Desarrollo de la innovación}

Para analizar la mejora en el aprendizaje de los estudiantes tras la implantación de las metodologías activas en la asignatura y la realización de las nuevas actividades, se han empleado las siguientes herramientas:

1) Encuesta inicial de diagnóstico. En la primera sesión de clase se pasó una encuesta anónima a los alumnos sobre los principales contenidos de la asignatura, muchos de los cuales eran totalmente nuevos para ellos. En la Tabla 1 se muestran dichos contenidos, indicando para cada uno si eran nuevos o si se habían tratado en asignaturas previas.

Tabla 1. Contenidos de la encuesta de diagnóstico

\begin{tabular}{|l|l|}
\hline Contenido & $\begin{array}{l}\text { Trabajado en asignatura previa } \\
\text { (curso/cuatrimestre) }\end{array}$ \\
\hline Identificación de riesgos & No \\
\hline Agentes físicos, químicos y biológicos (FQB) & No \\
\hline Ventilación y extracción & Sí (4\%/A) \\
\hline Almacenamiento & No \\
\hline Equipos de Protección Individual (EPIs) & Sí (3\%/A\&B y 4\%/A) \\
\hline Riesgo eléctrico & Sí (3\%/B y $\left.4^{\circ} / \mathrm{A}\right)$ \\
\hline
\end{tabular}

El tipo de preguntas realizadas sobre estos contenidos estaban dirigidas a identificar el conocimiento y compresión de los mismos, sin ningún tipo de aplicación o justificación, y valorar el nivel inicial de los alumnos tras trabajar alguno de estos conceptos en otras asignaturas previas en las que se ha incidido en la seguridad

2) Resultados académicos. Para analizar la evolución del aprendizaje de los alumnos en los contenidos incluídos en la encuesta inicial, se han comparado los resultados de ésta con los resultados finales obtenidos en distintos actos de evaluación, de entre los indicados en el

(cc) EY-NC-ND 2016, Universitat Politècnica de València

Congreso In-Red (2016) 
apartado de "Introducción" de este trabajo. Además, cabe distinguir dos niveles de evaluación, que serían los siguientes:

Nivel I. La evaluación del contenido se centra básicamente en el conocimiento y/o la comprensión del mismo. Sería, por tanto un tipo de evaluación análoga a la realizada en la encuesta inicial de diagnóstico.

Nivel II. La evaluación del contenido fue realizada de manera más compleja que el anterior nivel, incluyendo aplicación y pensamiento crítico en muchos casos.

En la Tabla 2 se indica la herramienta de evaluación empleada para analizar el grado de aprendizaje de cada contenido, junto con el nivel de evaluación aplicado. Para tener un orden de magnitud del trabajo realizado a lo largo de la asignatura en relación con cada contenido, en la Tabla 2 se han indicado también las actividades relacionadas con cada uno de ellos.

Tabla 2. Herramienta y tipo de evaluación de cada contenido

\begin{tabular}{|l|l|l|}
\hline Contenido & $\begin{array}{l}\text { Actividades } \\
\text { relacionadas }\end{array}$ & $\begin{array}{l}\text { Herramienta de evaluación } \\
\text { (Nivel de evaluación) }\end{array}$ \\
\hline Identificación de riesgos & $(1),(2),(3),(5)$ & $\begin{array}{l}\text { Examen (Nivel I) \& Informe } \\
\text { actividad (3) (Nivel II) \& Trabajo } \\
\text { final (Nivel II) }\end{array}$ \\
\hline $\begin{array}{l}\text { Agentes físicos, químicos y biológicos } \\
\text { (FQB) }\end{array}$ & $(1),(5)$ & Examen (Nivel I) \\
\hline Ventilación y extracción & $(2),(5)$ & Examen (Nivel I) \\
\hline Almacenamiento & $(4)$ & Trabajo final (Nivel II) \\
\hline Equipos de Protección Individual (EPIs) & $(2)$ & Examen (Nivel II) \\
\hline Riesgo eléctrico & $(2),(5)$ & Examen (Nivel I) \\
\hline
\end{tabular}

\section{Resultados}

A continuación se analizan los resultados recogidos a través de las herramientas comentadas en el apartado anterior.

1) Análisis de respuestas de la encuesta inicial

Para analizar las respuestas de la encuesta inicial se han contabilizado las mismas clasificándolas de acuerdo a los siguientes rangos:

- Blanco. Cuando la pregunta no se ha respondido.

- Incorrecta. Cuando la calificación de la pregunta (en base 1) ha sido inferior a 0,5.

- Bien. Cuando la calificación de la pregunta (en base 1) ha sido igual o superior a 0,5 , pero inferior a 0,75 .

(cc) EY-NC-ND 2016, Universitat Politècnica de València 
- Muy bien. Cuando la calificación de la pregunta (en base 1) ha sido igual o superior a 0,75 .

En la Fig. 1 se muestra el porcentaje de respuestas de cada rango para cada uno de los contenidos evaluados (ver Tabla 1).

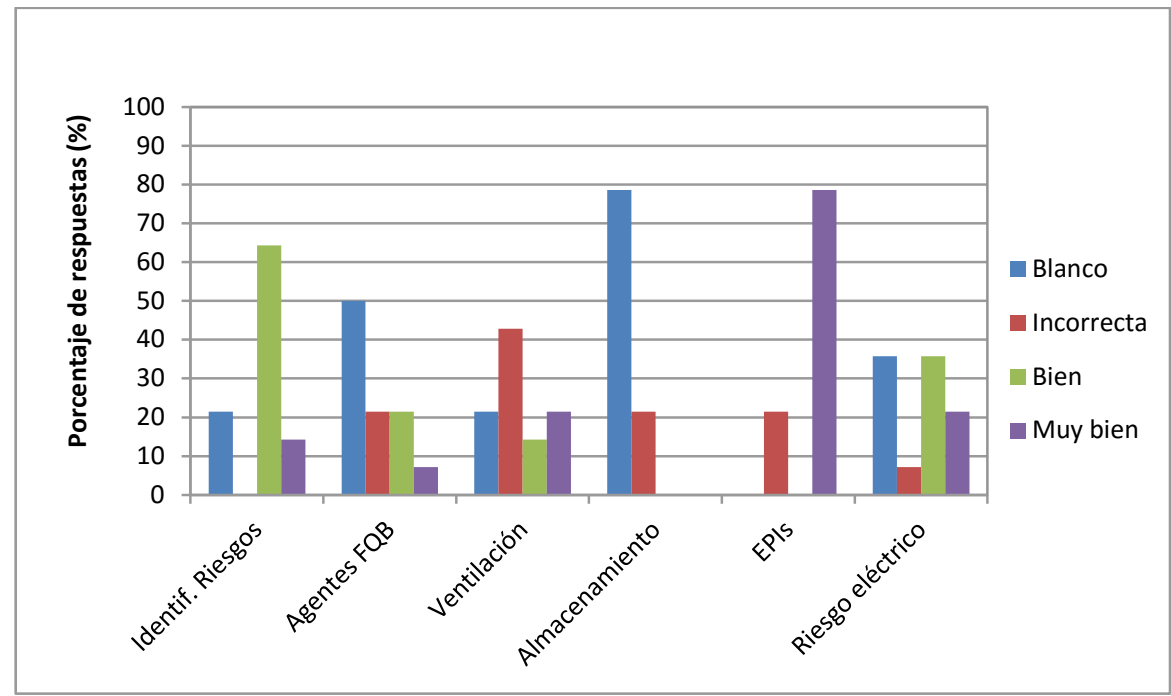

Fig. 1 Respuestas de cada contenido en la encuesta inicial de diagnóstico

Como se observa en laFig. 1, la pregunta sobre "Identificación de riesgos"fue respondida correctamente por la mayoría de los alumnos (64,3\% Bien y 14,3\% Muy bien), no habiendo ninguna respuesta Incorrecta, y habiendo 3 alumnos que no respondieron a la misma $(21,4 \%$ Blanco). Cabe destacar que se trataba de un contenido nuevo no trabajado específicamente en asignaturas previas, lo que muestra una buena intuición de los estudiantes respecto al mismo, probablemente por el trabajo general realizado en seguridad en otras asignaturas, aunque no se tratara específicamente este aspecto.

En las respuestas de la preguntas de "Agentes FQB", que también era un contenido nuevo para los alumnos, se observa mayor dispersión. La mitad de los estudiantes no respondieron a la pregunta (50\% Blanco), un 21,4 \% la respondieron Incorrecta, y el restante $28,5 \%$ lo hicieron correctamente (21,4\% Bien y 7,1\% Muy bien).

Respecto a la pregunta sobre "Ventilación", que era un contenido trabajado en el cuatrimestre anterior, se observa un mayor porcentaje de alumnos que han respondido (sólo $21,4 \%$ en Blanco), sin embargo, el porcentaje de respuestas Incorrecta es de un 42,9\%, significativamente superior al de respuestas correctas (14,3\% Bien y 21,4\% Muy bien). Parece, por tanto, que este contenido no ha quedado del todo claro o interiorizado por los estudiantes en la asignatura anterior, y requiere de un mayor refuerzo en esta asignatura. 
La pregunta sobre "Almacenamiento" fue la que obtuvo peores resultados de todas las de la encuesta inicial. Sólo un $21,4 \%$ de los estudiantes respondieron a la pregunta $(78,6 \%$ en Blanco), y además todos lo hicieron de manera Incorrecta. Este resultado era esperable ya que se trata de una pregunta que hacía referencia a un aspecto muy concreto del contenido.

En relación a la pregunta sobre EPIs, se observa que es la que produjo mejores resultados de toda la encuesta inicial, con un elevado porcentaje de respuestas por encima de 0,75 puntos (78,6\% Muy bien). Esto se puede explicar por el hecho de ser un contenido que se trabaja en varias asignaturas anteriores, tanto en el cuatrimestre inmediatamente anterior, como en las asignaturas experimentales de $3^{\text {er }}$ curso (ver Tabla 1 ).

Finalmente, la pregunta relativa a "Riesgo eléctrico" también presenta bastante dispersión de respuestas. Cabe señalar un procentaje significativo de respuestas correctas entorno al $57 \%$ (35,7\% Bien y 21,4\% Muy bien), y sólo un 7,1\% de respuestas Incorrecta entre las respondidas. Al tratarse de un contenido ya trabajado en asignaturas anteriores, estos resultados indican un adecuado grado de aprendizaje al respecto (superior al 50\% de los alumnos encuestados).

\section{2) Análisis de la evolución del aprendizaje}

En la Fig. 2 se muestran las calificaciones promedio (en base 1) alcanzadas por los estudiantes de la asignatura en las preguntas de la encuesta inicial relativas a cada contenido, y en la evaluación final de los mismos. Se han diferenciado en la gráfica las evaluaciones de nivel I y nivel II, de acuerdo a lo indicado en la Tabla 2, y, además, dentro del nivel II se ha distinguido el análisis del trabajo final de otras herramientas de evaluación (examen y/o informes).

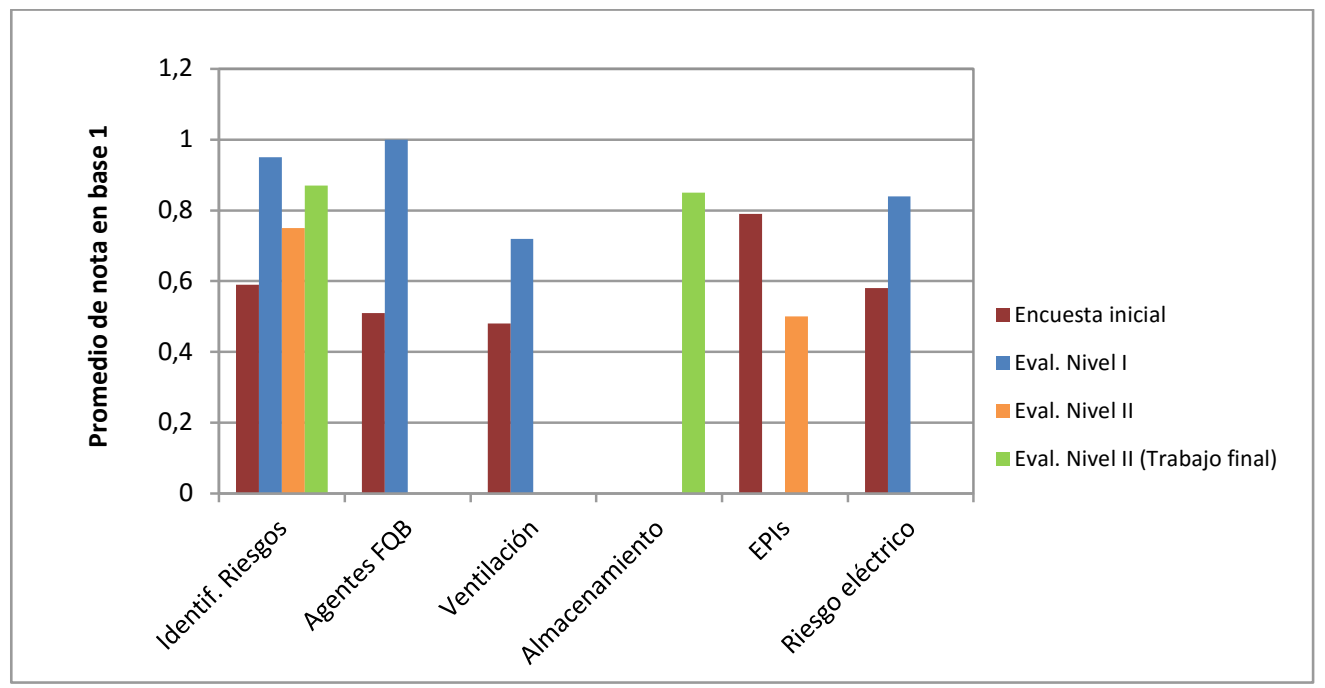

Fig. 2 Calificaciones promedio en cada contenido en la encuesta inicial y en la evaluación final 
A continuación se analiza el grado de mejora del aprendizaje en cada contenido, en base a los resultados mostrados en la Fig.2:

- "Identificación de riesgos". Este contenido fue evaluado de tres formas diferentes: en el examen final (nivel I) y a través del informe escrito y del trabajo final (nivel II)) Como se observa en la gráfica, en ambos niveles de evaluación se aprecia una mejora de la calificación promedio, respecto a la encuesta inicial. Dicha mejora es más significativa en el caso de la evaluación de nivel I por ser más sencilla, ya que sólo estaba referida a compresión; mientras que la de nivel II no mejora en tanta proporción ya que el tipo de evaluación implicaba análisis, reflexión y justificación. Sin embargo, cabe destacar los buenos resultados alcanzados en este segundo tipo de evaluación, y sobre todo la progresión en la calificación promedio que fue de 0,75 en base 1 en el informe escrito presentado en la actividad 3, y de 0,87 en base 1 en el trabajo final. Esta mejora está justificada principalmente por la práctica adquirida a lo largo de las distintas actividades, y por el feed-back proporcionado con la corrección del informe de la actividad 3.

- "Agentes FQB". Este contenido fue evaluado sólo con nivel I, a través de una pregunta de examen similar a la realizada en la encuesta incial. Se puede observar en la gráfica que la mejora en la calificación promedio fue muy significativa, pasando de una media de 0,51 a un promedio de 1 (ambos valores en base 1). Se trata, por lo tanto, de un contenido con una mejora del aprendizaje muy elevada.

- "Ventilación”. De forma análoga al contenido anterior, éste también se evaluó con nivel I, de manera muy similar a la encuesta. También en este caso se observa una mejora importante en la calificación promedio, que pasó de 0,48 a 0,72 en base 1, aunque no tan relevante como en el caso de "Agentes FQB". Los profesores de la asignatura pensamos que esto puede deberse a que hay varias actividades que indirectamente trabajan el contenido "Agentes FQB", mientras que el de "Ventilación" solo se trabaja en una. Esto podría mejorarse reforzándolo en varias actividades, lo cual se considerará para futuros cursos.

- "Almacenamiento". La mejora de este contenido es evidente, ya que se partía de un nivel nulo detectado en la encuesta inicial. Sin embargo, cabe destacar que el contenido se evaluó con un nivel tipo II a través del trabajo final, y que el promedio de calificaciones fue muy elevado $(0,85$ en base 1$)$. Creemos que esto ha sido favorecido por el hecho de haber realizado una actividad específica de trabajo guiado por el profesor en el aula del contenido.

- "EPIs". Este es el único contenido que ha empeorado en calificación promedio final ( 0,5 en base 1) respecto a la obtenida en la encuesta inicial ( 0,79 en base 1). Esto se debe, por un lado, al cambio en el modo de evaluación del contenido, ya que se evaluó con un nivel de tipo II en el examen, de manera muy diferente a la de la encuesta inicial en la que la pregunta estaba dirigida al simple conocimiento. Por otro lado, también puede haber influido el hecho de que haya sido el contenido trabajado en un menor número de actividades. 
- "Riesgo eléctrico". Este contenido fue evaluado con un nivel tipo I a través del examen, observándose una mejora significativa de la calificación promedio, que pasó de 0,58 en la encuesta inicial a 0,84 en la evaluación final.

Globalmente, se observa una mejora significativa del grado de aprendizaje de los alumnos en la asignatura, que pensamos que está favorecido por las nuevas metodologías implantadas y por las actividades llevadas a cabo, ya que los contenidos con mayor grado de mejora han sido aquéllos con actividades específicas o trabajados indirectamente a través de varias actividades.

\section{Conclusiones}

A la vista de los resultados analizados en este trabajo se puede concluir lo siguiente:

- En general, se detecta un buen resultado en la encuesta inicial en aquellos contenidos trabajado en asignaturas previas. Sin embargo, se ha identificado como contenido a reforzar el de "Ventilación", cuyos resultados en la encuesta inicial no han sido satisfactorios.

- Respecto a los contenidos nuevos evaluados en la encuesta inicial, aquellos más específicos han proporcionado gran número de respuestas en blanco. Sin embargo, se han detectado buenos resultados en algunos contenidos más intituitivos o trabajados indirectamente en asignaturas previas donde se aborda el tema de seguridad.

- Tras la implantación de las nuevas metodologías y la realización de las nuevas actividades, se ha observado una mejora significativa en el promedio de calificaciones de la mayoría de contenidos.

- La mejora detectada es mayor en aquellos contenidos que se trabajan a través de varias actividades o mediante una actividad específica diseñada exclusivamente para ello.

- Las mejoras en las calificaciones promedio han sido más elevadas cuando la evaluación realizada era relativa a conocimiento y compresión.

- Las evaluaciones que incluían análisis, reflexión y justificación no siempre han supuesto una mejora en las calificaciones al requerir un mayor nivel de aprendizaje y tratarse de conocimientos nuevos trabajados por primera vez. Por lo tanto, en los próximos cursos debería hacerse mayor hincapié en trabajar estas competencias a lo largo de la asignatura.

\section{Referencias}

ARAMENDI JAUREGUI, P., BUJAN VIDALES, K., GARÍN CASARES, S. y VEJA FUENTE, A. (2014). "Estudio de caso y aprendizaje cooperativo em la universidad" en Profesorado. Revista de currículum y formación del profesorado, Vol. 18 (1), pp. 413-429. 
BADIA, A. y GARCÍA, C. (2006). "Incorporación de las TIC en la enseñanza y el aprendizaje basados en la elaboración colaborativa de proyectos" en Revista de Universidad y Sociedad del Conocimiento, Vol. 3 (2), pp. 42-53.

BARBERA-RIBERA, T., ESTELLES-MIGUEL, C. y DEMA-PEREZ, C.M. (2015). "Student opinion on the application of active methodologies" en Peris-Ortiz, M., Merigó Lindahl, J. M. Sustainable Learning in Higher Education. Innovation, Technology and Knowledge Management. Switzerland: Springer International Publishing.

DARBRA, R. M., CASAL, J., PASTOR, E., VILCHEZ J. A., ARNALDOS, J. y PLANAS, E. (2012). "Risk analysis active learning through the investigation of real cases" en Process Safety and Environmental Protection, Vol. 90, pp. 430-435.

FERJENCIK, M. (2007). "Best starting point to comprehensive process safety education" en Process Safety Progress, Vol. 26 (3), pp. 195-202.

GARCÍA-FAYOS, B., SANCHO, M. y ARNAL, J. M. (2015). “Aplicación de metodologías activas de aprendizaje en una nueva asignatura de Grado en Ingeniería Química". Instituto de Ciencias de la Educación ICE-UPV. En: Congreso Nacional de Innovación Educativa y Docencia en Red IN-RED15 (julio, 2015, Valencia). Valencia: Editorial Universitat Politècnica de València.

GIL MONTOYA, C., BAÑOS NAVARRO, R., ALÍAS SÁEZ, A. y GIL MONTOYA, M. D. (2007). "Aprendizaje cooperativo y desarrollo de competencias" en JAC-07, $7^{a}$ Jornada sobre aprendizaje cooperativo. Valladolid. Disponible en $<$ http://157.88.123.53/JAC/GIAC_JAC/07/30.pdf $>$ [Consulta: 29 de marzo de 2016].

GRAELlS, M. y PÉREZ-MOYA, M. (2007) "Projecte PEEEQ: Planificació estratégica de les asignatures d'Experimentació en Enginyeria Química de l'Escola Universitària d'Enginyeria Tècnica Industrial de Barcelona". Universitat Politécnica de Catalunya, 2007.

PALAZÓN-PÉREZ DE LOS COBOS, A., GÓMEZ-GALLEGO, M., CÁNDICO GÓMEZGALLEGO, J. C., PÉREZ-CÁRCELES, M. C. y GÓMEZ-GARCÍA, J. (2011). "Relación entre la aplicación de metodologías docentes activas y el aprendizaje del estudiante universitario" en Bordón, Vol. 63 (2), pp. 49-58

SHALLCROSS, D. C. (2013). "Safety education through case study presentations" en Education for Chemical Engineers, Vol. 8, pp. e12-e30.

WESTRUP, U. y PLANANDER, A. (2013). "Role-play as a pedagogical method to prepare students for practice: The students' voice” en Högre utbildning, Vol. 3 (3), pp. 199-210. 